JORNADAS

\title{
MANEJO DE LA ALBUMINURIA. MOMENTO DE DERIVACIÓN AL NEFRÓLOGO
}

\author{
MANAGEMENT OF ALBUMINURIA. \\ MOMENT OF NEPHROLOGY REFERRAL
}

\author{
María José Pomares
}

\begin{abstract}
RESUMEN
En los últimos años surgieron nuevas observaciones que permitieron entender que el curso clínico de la enfermedad renal por diabetes podría ser diferente a la descripción tradicional. Uno de los primeros conceptos actuales es la recomendación de reemplazar el término de micro y macroalbuminuria por el de albuminuria, la cual se califica según su severidad en estadios A1, A2 y A3. La detección de la albuminuria como único marcador de enfermedad renal parece no ser suficiente para el diagnóstico temprano; es necesario además el monitoreo de la función renal usando ecuaciones de estimación del filtrado glomerular (FG). El control estricto de la glucemia, la presión arterial (PA) y el uso de IECA o ARA Il continúan siendo el tratamiento fundamental de la albuminuria. Para el control glucémico podría ser conveniente tener en cuenta los beneficios adicionales de los medicamentos antidiabéticos. La derivación al especialista en Nefrología debería plantearse de acuerdo al estadio de albuminuria y deterioro del FG.
\end{abstract}

Palabras clave: albuminuria, control de la glucemia, control de la presión arterial.

Revista de la Sociedad Argentina de Diabetes 2017; Vol. 51 (86-89)

\begin{abstract}
In the last years new observations arise that allowed to understand that the clinical course of kidney disease caused by diabetes might be different from the traditional description. One of the first current concepts is the recommendation of replacing the term micro and macroalbuminuria by albuminuria, which is classified according to its severity in stages A1, A2 and A3. The detection of albuminuria as single marker of kidney disease does not seem to be sufficient for the early diagnosis, it is also necessary the monitoring of the renal function using estimating equations of the glomerular filtration (GF). The strict monitoring of glycemia, blood pressure (BP) and the use of IECA or ARA // continue being the fundamental treatment of albuminuria. For glycemia monitoring, it may be convenient to consider the additional benefits of antidiabetic drugs. Nephrology referral should be considered according to the stage of albuminuria and GF deterioration.
\end{abstract}

Key words: albuminuria, glycemia monitoring, blood pressure control.

Revista de la Sociedad Argentina de Diabetes 2017; Vol. 51 (86-89)
Médica especialista en Nefrología, especializada en diabetes, Hospital Penna; Consultorios de Diabetes en Cereha S.A. y en DAS Obra Social del Congreso; miembro del Comité de Nefropatía de la Sociedad Argentina de Diabetes, CABA, Argentina

Contacto de la autora: María José Pomares

E-mail: mariajosepomares@live.com.ar
Correspondencia: Beruti $28286^{\circ}$ piso, Depto. A (C1425BBH), CABA, Argentina

Tel: (011) 4822-4272

Fecha de trabajo recibido: 18/08/17

Fecha de trabajo aceptado: 30/08/17

Conflictos de interés: la autora declara que no existe conflicto de interés

\section{Manejo de la albuminuria}

Durante varios años se describió el curso clínico característico de la nefropatía por diabetes (ND) como la aparición de microalbuminuria que progresaba a macroalbuminuria seguida de una disminución progresiva del filtrado glomerular (FG) y finalmente insuficiencia renal crónica (IRC), relacionada principalmente a daño glomerular y asociada a otras manifestaciones de daño microvascular como retinopatía por diabetes (RPD) ${ }^{1}$.
En relación al desarrollo de la ND, en los últimos años surgieron nuevas observaciones que permitieron entender que el curso clínico de esta patología puede en ocasiones ser diferente a esta descripción convencional. Uno de los primeros conceptos que surgió es la recomendación de reemplazar el término de micro y macroalbuminuria por el de albuminuria, que según su severidad serán estadios A1, A2 y A3 como se observa en la Tabla $1^{2}$. 


\begin{tabular}{|c|c|c|}
\hline $\begin{array}{c}\text { A1 } \\
\text { normal o } \\
\text { incremento leve }\end{array}$ & $\begin{array}{c}\text { A2 } \\
\text { incremento } \\
\text { moderado }\end{array}$ & $\begin{array}{c}\text { A3 } \\
\text { incremento } \\
\text { severo }\end{array}$ \\
\hline$<10$ ó $11-29 \mathrm{mg} / \mathrm{g}$ & 30 a $300 \mathrm{mg} / \mathrm{g}$ & $>300 \mathrm{mg} / \mathrm{g}$ \\
\hline
\end{tabular}

Tabla 1: Categorías de albuminuria. Índice albuminuria/creatininuria ${ }^{2}$.

Se observó que numerosos pacientes con diabetes mellitus (DM) tipo 1 y 2 presentan FG menor a $60 \mathrm{ml} / \mathrm{min}$ con normoalbuminuria, 24 y $51 \%$ respectivamente según los estudios Diabetes Control and Complications Trial (DCCT) y United Kingdom Prospective Diabetes Study (UKPDS) ${ }^{4}$. Por otro lado, datos surgidos de diferentes estudios contradicen la idea clásica de que la albuminuria indefectiblemente progresa a estadios de mayor severidad, de hecho se demostró que es más probable la regresión a valores normales o ninguna modificación de la misma que su progresión ${ }^{5,6}$. Por último, también se observó que la RPD puede no estar presente en pacientes con ND'.

Por lo descripto anteriormente, la estrategia actual de detección de la albuminuria como único marcador de enfermedad renal parece no ser suficiente para el diagnóstico temprano; es necesario además el monitoreo de la función renal usando ecuaciones de estimación del FG hasta tanto se cuenten con nuevos marcadores para ND ${ }^{1,5}$. Sin embargo, la presencia de albuminuria patológica y su progresión posterior tienen un valor predictivo significativo tanto para el riesgo de deterioro del $\mathrm{FG}^{7}$, las complicaciones cardiovasculares y la mortalidad, especialmente en $\mathrm{DM} 2^{6,8}$.

Las estrategias que se emplearon históricamente para la detección y tratamiento de la ND asumían implícitamente que el proceso subyacente de la enfermedad era sólo la glomeruloesclerosis por diabetes ${ }^{1}$. Clásicamente se describió la albuminuria de la ND como consecuencia casi exclusivamente de patología glomerular debida a la apoptosis y desprendimiento de podocitos, ensanchamiento de los pedicelos, alteración del citoesqueleto de actina, pérdida o modificación de la carga eléctrica de las proteínas de la hendidura de filtración y disfunción endotelial ${ }^{9}$. Actualmente también puede atribuirse un papel importante a la patología tubular en la patogenia de la albuminuria. Los altos niveles de glucosa, el aumento de la angiotensina II y el incremento de la expresión del factor de crecimiento transformante $\beta$ (TGF $\beta$ ) generan disfunción tubular y disminución de la reabsorción de albúmina y proteínas en el túbulo contorneado proximal (TCP) ${ }^{10,11,12}$.

En estos momentos el control estricto de la glucemia, de la presión arterial (PA) y el uso de IECA o ARA II continúan siendo el tratamiento fundamental de la albuminuria9,13. Se recomienda el uso de IECA o ARA II ante la presencia de hipertensión y/o albuminuria A2 ó A3 en DM. Se desaconseja la asociación de ambos o con bloqueantes de la renina por mayor frecuencia de eventos adversos ${ }^{5,14,15}$. El uso combinado con un antialdosterónico sería beneficioso ya que estas drogas evitan el escape de aldosterona que ocurre en hasta el $40 \%$ de los pacientes tratados con un IECA $5,13,15$. Además la aldosterona produciría una disminución adicional de la albuminuria por mecanismos moleculares que aún no están aclarados y serían independientes de su efecto sobre la disminución de la $\mathrm{PA}^{16}$. Otras terapias referidas al uso de estatinas/ fibratos ${ }^{9,13}$ activadores de la vitamina $D^{5,13,17}$ y fármacos para reducir la uricemia parecerían también tener efectos beneficiosos para la reducción de la albuminuria?.

Al momento de la elección de los fármacos para el control glucémico sería conveniente tener en cuenta los beneficios adicionales que se observan en algunos de ellos referidos a la reducción de la albuminuria por mecanismos independientes de su efecto sobre la disminución de la glucemia. Entre dichos fármacos, los inhibidores de SGLT-2 ejercerían su efecto sobre la ND a través de la reducción de la reabsorción de glucosa/sodio en el TCP y por lo tanto mayor aporte de sodio a la mácula densa en el túbulo distal 
causando, a través del mecanismo de retroalimentación tubuloglomerular, la vasoconstricción de las arteriolas aferentes con reducción de la hiperfiltración y la albuminuria ${ }^{18,19,20}$. Un mecanismo similar se propuso para las incretinas (DPP-4 y GLP-1) ya que el GLP-1 inhibe la acción del cotransportador de $\mathrm{Na}+/ \mathrm{H}+$ isoforma 3 (NHE3) en el TCP, aumentando la excreción de sodio y su aporte al túbulo distal $^{21,22}$. Además estas drogas también disminuirían la albuminuria por vías moleculares más complejas asociadas a disminución del estrés oxidativo, la inflamación y mejoría de la función endotelial ${ }^{23,24}$, mecanismos propuestos también para la disminución de la albuminuria que se observa en asociación a las tiazolidinedionas actuando a nivel de los receptores PPAR $\gamma^{14,20}$.

La bibliografía actual sugiere la derivación al especialista en Nefrología en caso albuminuria A2 con FG menor a $45 \mathrm{ml} / \mathrm{min}$, de FG menor a $30 \mathrm{ml} / \mathrm{min}$, albuminuria $\mathrm{A} 3$ persistente, ante progresión de la albuminuria en dos controles consecutivos o con valores de albuminuria próximos a $300 \mathrm{mg} / \mathrm{g}$, sedimento urinario con eritrocitos dismórficos, deterioro acelerado del FG, albuminuria en DM1 de reciente diagnóstico o sospecha de patología renal de otra causa $^{2}$. Podría sugerirse que ante la presencia de albuminuria menor a 300 mg/24 hs y FG normal, donde el control glucémico estricto es determinante para la evolución de la enfermedad renal, el seguimiento del paciente se lleve a cabo especialmente por el especialista en diabetes priorizando el control metabólico. Ante la caída del FG por debajo de $30 \mathrm{ml} / \mathrm{min}$ adquiere importancia el manejo por parte del especialista en Nefrología ya que deben contemplarse otros aspectos relevantes como anemia, alteraciones del medio interno y del metabolismo fosfocálcico. Dado que estas alteraciones aparecen más precozmente en los pacientes con DM ya con FG de $60 \mathrm{ml} / \mathrm{min}$ o menos, podría sugerirse que con $\mathrm{FG}$ entre 30 a $60 \mathrm{ml} / \mathrm{min}$ el seguimiento sea conjunto.

La prevalencia de la ND está aumentando con el tiempo a pesar de las recomendacio- nes ampliamente conocidas ${ }^{6}$. Para que las intervenciones resulten eficaces en la prevención de la misma deberían iniciarse mucho antes en el curso de la enfermedad, pero la albuminuria, hasta ahora el pilar para la detección precoz, parecería no ser suficientemente precisa para predecir el riesgo de ND. Una identificación de los pacientes que tienen DM y un alto riesgo de desarrollar ND podría revertir esta tendencia epidemiológica. Una cuidadosa evaluación de todos los parámetros actualmente disponibles, como historia familiar de enfermedad renal crónica, antecedentes de tabaquismo, sobrepeso u obesidad, evaluación de los niveles de lípidos y ácido úrico, monitorización de la PA, control estricto de la glucemia y especialmente mediciones periódicas de albuminuria y estimaciones del FG, pueden mejorar la capacidad para predecir y retrasar la ND, hasta tanto se cuente en la práctica diaria con nuevos marcadores para la misma ${ }^{13}$.

\section{BIBLIOGRAFÍA}

1. Kramer HJ, Nguyen QD, Curhan G, Hsu CY. Renal insufficiency in the absence of albuminuria and retinopathy among adults with type 2 diabetes mellitus. JAMA 2003; 289(24):3273-7.

2. KDIGO 2012. Clinical practice guideline for the evaluation and management of chronic kidney disease. Kidney Int. 2013; (3):1-163.

3. Molitch ME, Steffes M, Sun W, et al. Development and progression of renal insufficiency with and without albuminuria in adults with type 1 diabetes in the diabetes control and complications trial and the epidemiology of diabetes interventions and complications study. Diabetes Care 2010; 33(7):1536-43.

4. Retnakaran R, Cull CA, Thorne KI, Adler Al, Holman RR; UKPDS Study Group. Risk factors for renal dysfunction in type 2 diabetes: U.K. Prospective Diabetes Study 74. Diabetes 2006; 55:1832-1839.

5. Kim SS, Kim JH, Kim IJ. Current challenges in diabetic nephropathy: early diagnosis and ways to improve outcomes. Endocrinol Metab 2016; 31(2):245-53.

6. Halimi JM. The emerging concept of chronic kidney disease without clinical proteinuria in diabetic patients. Diabetes Metab 2012; 38(4):291-7.

7. Roscioni SS, Lambers Heerspink HJ, de Zeeuw D. Microalbuminuria: target for renoprotective therapy PRO. Kidney Int 2014; 86(1):40-9. 
8. Stephen R, Jolly SE, Nally JV Jr, et al. Albuminuria: when urine predicts kidney and cardiovascular disease. Cleve Clin J Med 2014; 81(1):41-50.

9. Kitada M, Kanasaki K, Koya D. Clinical therapeutic strategies for early stage of diabetic kidney disease. World J Diabetes 2014; 5(3):342-56.

10. Russo LM, Sandoval RM, Campos SB, et al. Impaired tubular uptake explains albuminuria in early diabetic nephropathy. J Am Soc Nephrol 2009; 20(3):489-94.

11. Tojo A, Kinugasa $S$. Mechanisms of glomerular albumin filtration and tubular reabsorption. International Journal of Nephrology 2012; 1-9.

12. Dickson LE, Wagner MC, Sandoval RM, Molitoris BA. The proximal tubule and albuminuria: really. J Am Soc Nephrol 2014; 25(3):443-53.

13. Kowalski A, Krikorian A, Lerma EV. Diabetic nephropathy for the primary care provider: new understandings on early detection and treatment. Ochsner $\mathrm{J}$ 2014; 14(3):369-79.

14. Fried LF, Emanuele N, Zhang JH, et al; VA NEPHRON-D Investigators. Combined angiotensin inhibition for the treatment of diabetic nephropathy. N Engl J Med 2013; 369(20):1892-903.

15. Quiroga B, Arroyo D, de Arriba G. Present and future in the treatment of diabetic kidney disease. J Diabetes Res 2015; 1-13.

16. MavrakanasTA, Gariani K, Martin PY. Mineralocorticoid receptor blockade in addition to angiotensin converting enzyme inhibitor or angiotensin II receptor blocker treatment: an emerging paradigm in diabetic nephropathy: a systematic review. Eur J Intern Med 2014; 25(2):173-6.
17. Agarwal R. Vitamin D, proteinuria, diabetic nephropathy, and progression of CKD. Clin J Am Soc Nephrol 2009; 4(9):1523-8.

18. Vallon $\mathrm{V}$, et al. Targeting renal glucose reabsorption to treat hyperglycaemia: the pleiotropic effects of SGLT-2 inhibition. Diabetologia 2016; 60 (2):215-225.

19. Fioretto $P$, Zambon $A$, Rossato $M$, et al. SGLT-2 inhibitors and the diabetic kidney. Diabetes Care 2016; 39(2):165-71.

20. Górriz JL, Nieto J, Navarro-González JF, et al. Nephroprotection by hypoglycemic agents: do we have supporting data? J Clin Med 2015; 4(10):1866-89.

21. Skov J. Effects of GLP-1 in the kidney. Rev Endocr Metab Disord 2014; 15(3):197-207.

22. Von Websky K, Reichetzeder C, Hocher B. Physiology and pathophysiology of incretins in the kidney. Curr Opin Nephrol Hypertens 2014; 23(1):54-60.

23. Haluzík M, Frolík J, Rychlík I. Renal effects of DPP-4 inhibitors: a focus on microalbuminuria. International Journal of Endocrinology 2013; 7. Panchapakesan U, Mather A, Pollock C. Role of GLP-1 and DPP-4 in diabetic nephropathy and cardiovascular disease. Clin Sci. 2013; 124(1):17-26.

24. Caramori ML, Fioretto $P$, Mauer M. Enhancing the predictive value of urinary albumin for diabetic nephropathy. J Am Soc Nephrol 2006; 17(2):339-52. 